Meta

Journal des traducteurs

Translators' Journal

\title{
Traduire l'humour dans des films français doublés en espagnol
}

\section{Pedro Mogorrón Huerta}

Volume 55, numéro 1, mars 2010

Le parcours du sens : d'une langue à l'autre — Mélanges offerts à André Clas

The Way of Meaning: From a Language to Another - Collection of Articles Offered to André Clas

URI : https://id.erudit.org/iderudit/039603ar

DOI : https://doi.org/10.7202/039603ar

Aller au sommaire du numéro

\section{Éditeur(s)}

Les Presses de l'Université de Montréal

ISSN

0026-0452 (imprimé)

1492-1421 (numérique)

Découvrir la revue

Citer cet article

Mogorrón Huerta, P. (2010). Traduire l'humour dans des films français doublés en espagnol. Meta, 55(1), 71-87. https://doi.org/10.7202/039603ar

\section{Résumé de l'article}

L'humour est un phénomène universel, présent dans toutes les cultures, les langues et les civilisations, et très difficile à traduire. Lorsque la traduction d'un élément humoristique doit, de plus, se faire dans un texte audiovisuel, la difficulté augmente considérablement, en raison des caractéristiques et des restrictions inhérentes à ce type de support qui est subordonné aux limitations temporelles et spatiales des scènes et des dialogues. Dans le présent article, nous analysons l'humour dans trois films français illustrant les catégories de la comédie ou du drame, ainsi que dans leur adaptation en espagnol. Nous analysons différentes situations dans lesquelles apparaissent des éléments humoristiques, ainsi que les moyens utilisés pour les transmettre. Nous nous demandons si la version doublée reproduit cet humour de façon intégrale. Bien que notre analyse se limite à deux langues, la méthode utilisée devrait pouvoir s’appliquer à de nombreuses autres. 


\title{
Traduire l'humour dans des films français doublés en espagnol
}

\author{
PEDRO MOGORRÓN HUERTA \\ Universidad de Alicante, Alicante, Espagne \\ pedro.mogorron@ua.es
}

\begin{abstract}
RÉSUMÉ
L'humour est un phénomène universel, présent dans toutes les cultures, les langues et les civilisations, et très difficile à traduire. Lorsque la traduction d'un élément humoristique doit, de plus, se faire dans un texte audiovisuel, la difficulté augmente considérablement, en raison des caractéristiques et des restrictions inhérentes à ce type de support qui est subordonné aux limitations temporelles et spatiales des scènes et des dialogues. Dans le présent article, nous analysons l'humour dans trois films français illustrant les catégories de la comédie ou du drame, ainsi que dans leur adaptation en espagnol. Nous analysons différentes situations dans lesquelles apparaissent des éléments humoristiques, ainsi que les moyens utilisés pour les transmettre. Nous nous demandons si la version doublée reproduit cet humour de façon intégrale. Bien que notre analyse se limite à deux langues, la méthode utilisée devrait pouvoir s'appliquer à de nombreuses autres.
\end{abstract}

\begin{abstract}
Humour is a universal and cultural phenomenon very difficult to translate. Furthermore, as far as audiovisual texts are concerned, the degree of difficulty increases considerably because of their inherent characteristics, such as time or space limitation in dialogs and scenes. In this article we analyze the translation strategies of humour patterns used in the Spanish adaptations of three French films belonging to the comedy or drama categories. We stress the importance of translation strategies used in order to prevent the loss of potentially humorous elements. Even though our analysis is performed with only two languages, our methodology can also be applied to many others.
\end{abstract}

\section{MOTS-CLÉS/KEYWORDS}

traduction audiovisuelle, doublage, humour

audiovisual translation, dubbing, humour

Le présent article fait état d'une analyse de différentes situations humoristiques observées dans des films français et dans leurs versions espagnoles doublées. Étant donné que les principaux moyens humoristiques sont visuels et verbaux, nous nous concentrerons sur les procédés de doublage et laisserons de côté le sous-titrage. L'étude des procédés humoristiques utilisés dans les originaux et les doublages est circonscrite à trois films français très connus. Il s'agit de deux comédies, dans lesquelles abondent les situations comiques, et d'un drame, respectivement:

- Les visiteurs: Ils ne se sont pas nés d'hier (1993)';

- Le dîner de cons $(1998)^{2}$;

- La haine (1995) $)^{3}$.

Nous avons comparé deux comédies et un drame afin de mettre en relief les procédés humoristiques dans des genres différents et leur caractère universel. Les 
éléments humoristiques apparaissant dans différentes scènes de ces trois films ont été classés et leur traduction en espagnol a été analysée.

\section{La traduction de l'humour dans les productions audiovisuelles}

Bien que l'essentiel des études de traduction porte sur la traduction écrite, les trente dernières années ont vu l'apparition de nombreux travaux (articles, ouvrages, thèses) traitant de traduction audiovisuelle. Il existe, par contre, peu de travaux traitant de la traduction de l'humour dans les productions audiovisuelles (Zabalbeascoa 2001; Bernal Merino 2002; Díaz Cintas 2003), sans doute en raison des difficultés soulevées. Selon Zabalbeascoa (2001 : 251) : «Para entender la traducción del humor en los textos audiovisuales es necesario conocer los factores propios de la traducción, por un lado, del humor, por otro, y de los textos audiovisuales, por otro ${ }^{4}$. " En effet, la qualité des traductions de l'humour dans les productions audiovisuelles ne peut être obtenue par la seule analyse des aspects linguistiques: le traducteur devra également connaître en profondeur la culture et la société du pays dans lequel a été réalisée la version originale du film et du pays cible. Ceci, afin de reproduire les situations ou les adapter à la mentalité et à la culture du public destinataire, opérations très difficiles, car, comme on le sait, l'humour est particulièrement lié aux racines culturelles caractéristiques des différentes sociétés. Ainsi, de nombreuses productions d'Espagne ou des États-Unis n'ont pas le même succès dans les pays d'Amérique du Sud ou au Royaume-Uni, et inversement, bien que le public utilise une langue commune dans les deux cas. Pour garantir le succès de certains films européens outre-Atlantique, il n'est pas rare que des «remakes» voient le jour. Ainsi, Trois hommes et un couffin, réalisé par Coline Serreau $(1985)^{5}$ a donné lieu à Three men and a baby de Leonard Nimoy $(1987)^{6}$, et La cage aux folles, d'Édouard Molinaro $(1978)^{7}$ a donné The birdcage $(1996)^{8}$. Étant donné la nature multisémiotique des documents audiovisuels, il faudra inévitablement tenir compte du fait que l'unité de sens résulte de l'union des dialogues, issus d'un texte écrit, et des images, qui «traduisent» l'interprétation des acteurs. Souvent, donc, l'image sert de support au dialogue grâce à la gestuelle des acteurs qui utilisent cette dernière pour obtenir un effet comique, comme le rappelle Díaz Cintas (2003: 256): «Recordemos aquí que muchas situaciones de humor se apoyan tanto en el soporte lingüístico como en la actuación gestual del personnaje ${ }^{9} »$. Finalement, comme nous avons limité notre analyse aux versions doublées, de nombreuses scènes exigeront une synchronie temporelle et labiale.

\section{Repérage des éléments humoristiques}

Tous les éléments tels que dialogues, marques culturelles, particularités de langue (variétés diatopiques, diachroniques, jeux de mots, etc.) et images, vont donc être très importants pour réaliser une bonne traduction de l'humour dans le cadre d'une production audiovisuelle. Zabalbeascoa (2001 : 258-260) propose une des classifications les plus complètes qui aient été réalisées sur le type d'humour dans la traduction audiovisuelle en relation avec les problèmes de traduction. Il distingue ainsi :

- la blague internationale (chiste internacional);

- la blague culturelle-institutionnelle (chiste cultural-institucional);

- la blague nationale (chiste nacional); 
- la blague linguistique (chiste lingüístico-formal);

- la blague non verbale (chiste no verbal);

- la blague paralinguistique (chiste paralingüístico);

- la blague complexe (chiste complejo).

L'humour est sans aucun doute un phénomène universel, présent dans toutes les cultures, les langues et les civilisations, qui a pour objectif principal de distraire et de faire rire en soulignant le comique ou le ridicule dans la vie quotidienne. Il est très difficile de le définir en raison des différents genres humoristiques existants et des contenus culturels propres à différents groupes se distinguant sur les plans linguistiques, géographiques ou sociaux. De plus, il est souvent subjectif, car ce qui est drôle ou risible dans une culture ou un pays ne produit pas nécessairement le même effet dans une autre culture ou un autre pays, voire chez une autre personne du même groupe. Ces différents paramètres font que la traduction de l'humour est sans aucun doute des plus complexes, car le traducteur, dans sa recherche de l'équivalence, doit essayer de reproduire dans le texte cible le même effet humoristique que celui qui est véhiculé par l'original, afin que le lecteur puisse en apprécier toutes les subtilités. Mais si, en plus, la traduction d'un élément humoristique (blague, histoire drôle, mot d'esprit, jeux de mots, etc.) doit se faire dans un texte audiovisuel, la difficulté augmente considérablement, étant donné les caractéristiques et les restrictions inhérentes à ce support. En effet, il est toujours subordonné aux limitations temporelles et spatiales des scènes et des dialogues ainsi que, fréquemment, à la synchronisation labiale.

Après le visionnement des trois films et l'analyse des scènes dans lesquelles nous avons observé la présence d'aspects humoristiques, nous avons adopté une classification fondée sur le mode d'expression de l'humour par le texte ou par l'image:

- expression visuelle;

- expression visuelle renforcée par le texte;

- expression dans le texte.

Ainsi, selon Chaume (2004: 186), "[l]os textos audiovisuales constituyen un género paradigmático en el cual la información no verbal desempeña un papel sumamente relevante. Precisamente aquello que nos permite agrupar estos textos en un género paradigmático, o género de géneros, es que en casi todos ellos el significado emana de la interacción entre información verbal e información no verbal ${ }^{10}$ ».

\subsection{L'expression visuelle de l'humour}

L'humour s'exprime visuellement lorsqu'il passe par l'image, c'est-à-dire par l'interprétation des acteurs, dans le cadre d'une situation comique par elle-même, sans l'aide des dialogues. Il est alors possible de transposer dans de nombreux pays ou de nombreuses cultures différentes les signes non verbaux, images et interprétation gestuelles des acteurs. Comme l'expression universelle bien connue le souligne, une image vaut mille mots. L'image sert, dans de nombreux cas, de catalyseur pour provoquer un sourire ou un éclat de rire, par la mise en scène de situations rocambolesques, loufoques ou ridicules, comme des chutes, ou par des regards d'incrédulité, etc. Ainsi, dans l'exemple (1) extrait du film Le dîner de cons, un homme élégamment 
habillé (costume, cravate) s'amuse à lancer des boomerangs dans un parc, son téléphone portable sonne. Il lance un boomerang, que sa conversation lui fait oublier, mais le boomerang revient évidemment à son point de départ et le frappe en plein visage, provoquant sa chute... et l'hilarité du spectateur!

(1) - Mais c'est épatant, ça, aucun problème Monsieur Michaud, je serai avec vous mercredi sauf incident... hoooo.

(Le dîner de cons)

- Estupendo sí, ningún problema Sr Michaud le veré el miércoles a menos que surja algún...oooooh.

(La cena de los idiotas)

Dans l'exemple (2) de Les visiteurs, Godefroy de Montmirail, un noble du XII siècle envoyé par erreur dans le $\mathrm{xx}^{\mathrm{e}}$ siècle par un mage qui se trompe de formule magique, et son valet Jacquouille prennent un bain avec leurs sous-vêtements moyenâgeux, qui sont tellement sales qu'ils en noircissent l'eau. Ils jettent dans la baignoire tous les onguents (paillettes de savon, crème, savon, etc.) qu'ils peuvent trouver dans la salle de bain. Godefroy va même jusqu'à vider sur lui un énorme flacon de Chanel $n^{\circ} 5$, ce qui suscite chez le spectateur un fou rire... ou une exclamation d'horreur!

(2) - Qu'est-ce qu'ils ont nos pieds, messire?

- Je n'en sais rien, ce gueux finasse sans arrêt... Ah! Ouh! c'est bouilli... Allez, mets les onguents!

(Godefroy et Jacquouille versent dans le bain tout ce qu'ils trouvent autour de la baignoire pendant une vingtaine de secondes.)

Jacquouille, frotte-moi!

(Les visiteurs)

- ¿Qué les pasa a nuestros pies?

- No lo sé, otro plebeyo enriquecido. Ahhh, está hirviendo... muy bien los ungüentos...

¡Delcojón frótame!

(Los visitantes)

L'exemple (3) est tiré de La haine, film qui dénonce la marginalisation des jeunes descendants d'immigrés qui habitent dans les cités en France. Il met en scène trois jeunes ayant des problèmes d'identité et d'intégration, Saïd, Hubert et Vinz. Après avoir passé une journée à se battre contre tout et n'importe quoi représentant la société en général, ils finissent leur journée dans une exposition d'art moderne où l'artiste colle des objets d'apparence très enfantins dans des tableaux.

(3) - C'est affreux, affreux, affreux, affreux, affreux, affreux.

- Hubert... il est connu le mec qui fait ça?

- Il sera connu quand il aura dix-huit ans.

(La haine)

- ¡Esto de aquí es una mierda como un piano!

- Hubert...jes famoso el que hace esto?

- Este será famoso cuando cumpla 18 años.

(El odio)

Le contraste entre, d'un côté, la vie problématique des jeunes marginaux confrontés à de nombreux problèmes et à une vie sans futur et, de l'autre, ce genre 
d'art fait sourire, à tout le moins, le spectateur. Évidemment, il s'agit d'un autre genre d'humour, plus corrosif, moins hilarant, associé à une critique et à une dénonciation de la société, mais il s'agit quand même d'humour.

Dans cette première catégorie, illustrée par les exemples (1) à (3), ce sont des signes non verbaux qui expriment l'humour. Le dialogue n'est pas dominant, car le rire est tout d'abord produit par la situation et l'interprétation des acteurs. C'est la force des images qui exprime le contenu humoristique, lequel n'a pas nécessairement besoin de s'appuyer sur des mots. On pourrait changer une grande partie ou la totalité du dialogue, voire l'éliminer totalement sans aucun problème. La traduction des échanges verbaux sera donc, généralement, littérale et ne présentera pas de difficultés, car ce sont les gestes, les bruits, les onomatopées, les images qui expriment l'effet humoristique indépendamment du pays ou de la culture d'accueil.

\subsection{L'expression visuelle de l'humour renforcée par le texte}

Dans cette catégorie de situations, l'humour exprimé par les images est renforcé cette fois par un dialogue. Il s'agit, pour nous, du procédé prototypique de production audiovisuelle de l'effet humoristique, qui résulte de l'union de deux modes distincts de communication. Le résultat est une augmentation de l'expressivité du message et de la scène et, par conséquent, de l'effet recherché chez le spectateur.

Dans l'exemple (4), Godefroy de Montmirail apprend que son château n'appartient plus à sa famille. Il promet alors à sa petite-petite-petite-petite-fillotte (Béatrice) qu'il va le lui racheter et que, dans le cas contraire, il étripera son propriétaire actuel (en fait le descendant de son valet, Jacquouille...). Il chante alors avec Jacquouille une sorte de chanson cruelle, tout en s'esclaffant, tandis que les hôtes, Béatrice et son mari Pierre-Henri, restent ahuris et stupéfaits.

(4) - Pardonnez ce marouffle, mais il est si triste d'apprendre qu'un gueux possède Montmirail!

- Je ne vois pas pourquoi.

- Ma douce et lumineuse fillotte, je n'aurai de cesse de réparer cette infamie. Ce Jacquard va vous rendre le château contre écus sonnants et trébuchants et s'il refuse, je l'étripe.

- Et on lui pèlera le jonc comme au bailli du Limousin...

- (Jacquouille et Godefroy ensemble) ... qu'on a fendu un beau matin, qu'on a pendu, avec ses tripes.

(Les visiteurs)

- Perdonad a este patán, más está muy triste al saber que un plebeyo posee Miramonte.

- No veo por qué.

- Mi dulce y radiante hijita, no cejaré hasta reparar esta infamia. Ese Delculón os devolverá el castillo a cambio de un puñado de escudos y si se niega lo destripo.

- Le daremos mucha caña como al duque de Champaña...

- (Delcojón y Godofredo juntos) al que rajamos con saña... y ahorcamos con mucha maña.

(Los visitantes)

Dans la version espagnole, le message est rendu, mais on note de nombreuses différences de forme, d'adaptations et de naturalisation d'éléments. Une rime dans deux vers de la version française (Limousin, matin, tripes) est remplacée par une rime 
de trois vers (Champaña, saña, maña); le traducteur a changé l'origine du bailli, le Limousin, pour la Champagne (Champaña). Il faut souligner ce gain du traducteur, toutefois, la chanson française est beaucoup plus agressive que celle de la version doublée. C'est ce contenu, que nous pourrions qualifier de sanguinaire, qui, associé à l'expression des visages des deux hommes ainsi qu'à la surprise et l'effroi de Béatrice et de son époux, provoque le rire du spectateur.

L'exemple (5) met en scène Pierre Brochant, éditeur, qui se moque de ceux qu'il qualifie de cons. Ceux-ci sont invités à des soupers pour être la risée de son groupe d'amis. Il a quelques problèmes avec sa victime du moment, François Pignon (nous sommes en présence du thème bien connu du moqueur ridiculisé ${ }^{11}$ ), et attend la visite d'un ami de ce dernier, censé lui apporter l'adresse d'un homme avec lequel sa femme le trompe. L'ami en question est inspecteur des impôts, et il est à craindre que le luxe dans lequel vit Brochant attise ses soupçons. Le seul vin disponible dans la maison étant un Château Lafite Rothschild 1978, l'inspecteur des impôts pourrait soupçonner un train de vie très élevé.

(5) (Pierre Brochant prend dans une armoire une bouteille sur laquelle on peut lire la mention vinaigre.)

- Il a du nez hein!

- Qu'est-ce que tu fais?

- Je fous du vinaigre dans mon Château Lafite! C'est un truc que je te donne si tu veux transformer un très grand vin en piquette! Et voilà! Le gros Lafite qui tache!... Goûte!

- Ah non, non. Goûte toi-même!

- Non merci!

(Pierre goûte le vin.)

- Alors?

- Ah c'est bizarre, ça lui donne du corps, je trouve!

- Merde alors!

- Il est pas plus mauvais... Il serait même plutôt meilleur.

- Nettement!

- Faites voir!

(Le dîner de cons)

(Coge una botella en la que hay escrito: vinaigre).

- Hum, tiene aroma.

- ¿Qué haces?

- Añado vinagre al Château Lafite, un truco ideal para transformarlo en un vino mediocre. Ya está adios al gran Lafite.

- Prueba.

- ¡No, no, tú primero!

- iNo, no gracias!

- ¡Y bien!

- ¡Es curioso, aún tiene más cuerpo!

- ¡Tienes razón!

- ¡No lo ha estropeado! Yo diría que lo ha mejorado.

- Estoy de acuerdo.

- ¿Puedo?

(La cena de los idiotas)

Cette scène qui ridiculise le snobisme de certains connaisseurs a un effet humoristique garanti. Les images seules permettent certes de suivre le fil de l'action grâce 
à des icônes expressives comme le gros plan sur l'étiquette de la bouteille de vinaigre, mais le dialogue renforce le contenu des images, notamment par la mention d'un vin qui vaut des centaines d'euros.

(6) - Il s'appelle Juste Leblanc.

- Ah bon, il n'a pas de prénom?

- Je viens de vous le dire: Juste Leblanc... Leblanc, c'est son nom, et c'est Juste son prénom.

- Hmm...

- Monsieur Pignon, votre prénom à vous, c'est François, c'est juste?

- Oui.

- Eh bien lui, c'est pareil: c'est Juste!

(Le dîner de cons)

- Se llama Solo Leblanc.

- ¡Ah! ¿no tiene nombre?

- Ya se lo he dicho, Solo Leblanc... Leblanc es el apellido, y Solo es el nombre.

- ¡Aah!

- Sr Piñón, su nombre es François, ¿cierto?

- Si.

- ¡Pues el de Leblanc, es Solo!

(La cena de los idiotas)

Le jeu de mots original sur le prénom Juste ( $\underline{\text { Solo })}$ est renforcé par l'interprétation magistrale de Jacques Villeret (dans le rôle de Pignon), dont l'air ahuri provoque le rire du spectateur, mais n'est pas rendu directement en espagnol.

Ces deux premières situations font appel à un humour bateau, commun à de nombreux pays. Ils jouent sur un comique de situation ou de caractère plus ou moins transposables d'un pays à l'autre. Dans les deux cas, l'interprétation gestuelle et orale des acteurs est fondamentale pour susciter une bonne partie du comique de la situation, même si le talent des acteurs de doublage n'est pas toujours à la hauteur de celui des acteurs d'origine.

\subsection{L'expression humoristique dans le texte}

Contrairement aux exemples analysés plus haut, certaines productions audiovisuelles, comme les films de Louis de Funès, du type Le gendarme de Saint-Tropez ou La soupe aux choux, ou, en anglais, celles des Monty Python se caractérisent par des dialogues qui deviennent prédominants dans l'expression de l'humour qui est alors plus complexe, plus fin, plus élaboré que celui véhiculé par les images. Le dialogue est une source essentielle d'informations que la traduction devra rendre. De nombreux films visent à attirer un large public national, bien caractérisé linguistiquement et culturellement, en lui transmettant de nombreux messages, sociaux, ethniques, en reproduisant une culture et une expérience commune, qui leur permettra de comprendre dans le cas de l'humour, les blagues, les jeux de mots et qui donnera beaucoup de mal aux traducteurs pour transcrire ces contenus dans une autre langue. Voyons l'exemple (7), tiré des Visiteurs:

(7) - Quelle excitation de pouvoir toucher et baiser sa petite fillotte!

- Il est excité par quoi? Il veut baiser qui? 
- ¡Qué excitación poder besar y tocar a mi hijita!

- ¿Qué dice que le excita? ¿Qué quiere hacerle?

(Los visitantes)

L'humour est provoqué par le double sens du verbe baiser qui, au XII ${ }^{\mathrm{e}}$ siècle, s'utilisait dans le sens: "appliquer, poser sa bouche sur le visage, la main ou sur une partie du corps de (qqn) par affection, amour, respect $»^{12}$, tandis qu'à partir du $\mathrm{XVI}^{\mathrm{e}}$ siècle est apparu le sens de: "posséder sexuellement une femme». Ce double sens est bien difficile à rendre dans une autre langue par un seul mot ou une seule expression en respectant les limites de temps et la synchronie. De fait, dans la traduction en espagnol, le jeu de mots est perdu, mais le public hispanophone comprend tout de même la réaction de Pierre-Henri.

La difficulté d'exprimer dans un doublage l'humour contenu dans un texte se retrouve dans de nombreuses situations. L'analyse de différents contextes nous a permis de déterminer plusieurs sous-catégories.

\subsubsection{Humour fondé sur des modalités linguistiques}

Dans Les visiteurs, l'un des ressorts de l'humour est lié à l'usage de différences linguistiques diachroniques, de nature lexicale, entre le français du Moyen-Âge et le français moderne: tensions liées à l'évolution du sens (exemple [7]), usage de formes anciennes (exemple [8]) ou reprise de mots tombés en désuétude (exemples [9] et [10]).

Ainsi, dans l'exemple (9), le film utilise les formes en -ois (anglois) de l'ancien français, qui dans de nombreux cas sont devenus -ais au XVIII ${ }^{\mathrm{e}}$ siècle, d'où l'usage actuel anglais $^{13}$.

(8) - Messire Godefroy, les Anglois, les Anglois.

(Les visiteurs)

- Sire Godofredo, los Ingleses, los Ingleses.

(Los visitantes)

En français, au $\mathrm{XVI}^{\mathrm{e}}$ siècle, le mot réussissement ${ }^{14}$ cohabitait avec la forme réussite (logro) qui a fini par s'imposer (exemple [9]).

(9) - Mais tu es mon fillot, je t’aime, je suis fier de ton réussissement.

(Les visiteurs)

- ¡Pero tú eres mi hijito, te quiero! Estoy muy orgulloso de tus logros.

(Los visitantes)

Le terme brossoir, "petite brosse pour nettoyer les pièces sorties des moules ${ }^{15}$ ", très peu connu du grand public, a été employé dans le film de par sa ressemblance avec brosse et sa sonorité ancienne [10]).

(10) - Ah, quel étrange brossoir!

- Qui fera bien l'affaire.

(Les visiteurs)

- ¡Qué extraño cepillo!

- Hará su trabajo... 
Bien que, dans certains dialogues, le traducteur soit parvenu à utiliser des tours linguistiques en espagnol rappelant le Moyen-Âge, le procédé n'est cependant pas généralisé. Très fréquemment, comme dans les exemples (8) à (10), la référence a été perdue, ce qui entraîne une perte d'effet humoristique.

Certains effets humoristiques s'appuient, chez certains personnages, sur des différences de niveau de langue liées à une appartenance socioculturelle particulière, qui se traduisent par de nombreuses incorrections linguistiques. Dans l'exemple (11), le jeune Saïd utilise une concordance de temps incorrecte, l'indicatif au lieu du subjonctif qu'il vienne me voir.

(11) - Eh, toi, dis à ton frère qu'il vient me voir... Eh, dis-lui qu'il vient me voir. Et c'est pas mortel comme phrase, ça! Le mec, il lui dit, moi je te tue gratuit.

(La haine)

- Oye dile a tu hermano que venga a verme... Díle que venga a verme. No es una frase genial!... A ti te mato gratis!

(El odio)

Dans l'exemple (12), Ginette (la clocharde dont Jacquouille tombe amoureux) fait le même genre d'erreur:

(12) - Parce qu'en fait j'ai une formation de danseuse chanteuse, eh ben $\underline{\text { si vous puissiez }}$ m’avoir une petite audition, hé ben, ça me changerait la vie!

(Les visiteurs)

- No en serio tengo estudios de danza y de canto y si pudieran conseguirme una pequeña audición eso me cambiaría la vida.

(Los visitantes)

Ce type d'erreur marque une absence d'éducation suscitant l'hilarité. Dans les deux cas, le traducteur n'a pas reproduit ces erreurs, qui deviennent alors imperceptibles pour le public hispanophone. L'incorrection de l'exemple (12) aurait pourtant pu être reproduit par si podieséis aconseguirme.

\subsubsection{Humour culturel}

Toute production audiovisuelle comprend de nombreuses références extralinguistiques connues et utilisées par le public d'origine. Ces références culturelles font partie de la vie quotidienne et peuvent se rapporter à une infinité de sujets (vie culturelle, musique, cinéma, télé, gastronomie, religion, goûts, coutumes, marques commerciales, etc.), d'autant plus difficiles à rendre dans la traduction qu'ils sont éloignés de la culture à laquelle appartient le traducteur.

De nombreux gags se fondent sur les références culturelles nationales, par exemple le football, le vin (voir l'exemple [5]), des personnalités connues.

\subsubsection{Le football}

L'exemple (13) contient de nombreuses références culturelles, rendues de façon variable en espagnol.

(13) - Alors monsieur l'Auxerrois?

- Aux chiottes l'OM, aux chiottes l'OM, aux chiottes!

- Tu vas voir mercredi, Auxerres... 
- Como está ese hincha..

- ¡Abajo Olimpic, abajo Olimpic, abajo!

- Vamos a daros una paliza...

(La cena de los idiotas)

L'apostrophe Monsieur l'Auxerrois fait référence au fait que François Pignon est un supporter de l'équipe de football relativement modeste de la ville d'Auxerres, moins connue que l'Olympique de Marseille (l'OM). Cette équipe n'étant pas très connue en Espagne, le traducteur a utilisé le terme hincha qui fait référence aux supporters en général. Aux chiottes l'OM, aux chiottes l'OM, aux chiottes! est chanté sur l'air bien connu en France pour, entre autres, encourager la sélection nationale de football: Allez les bleus... Tous les Français savent que l'OM est l'Olympique de Marseille. En traduisant par Olimpic, le traducteur a tenté de compenser la perte par une appellation plus générique. Enfin, le français est beaucoup plus vulgaire (et expressif) que la traduction espagnole: Abajo Olimpic ( $A$ bas l'Olympique).

\subsubsection{Célébrités}

Les références à des personnalités très connues dans une culture donnée constituent un moyen humoristique fréquemment utilisé, comme dans les deux exemples suivants $(14,15)$.

(14) - Est-ce que des fois vous connaissez pas, heu, Eddie Barclay?

$-[\ldots]$

- Ben quoi chochotte... J'chante pas plus mal que Stéph' de Monac'...

(Les visiteurs)

- ¿No conocen por casualidad a un productor?

$-[\ldots]$

- Qué pasa colegas, no canto peor que Stefi de Mónaco...

(Los visitantes)

(15) - Tu te rappelles quand même de ton nom?

- Il ne se rappelle de rien. Il est complètement amnésique. Il sait même pas qui est Michel Drucker.

- Amnésique, mais le pauvre!

(Les visiteurs)

- ¿Y de tu nombre, te acuerdas?

- No se acuerda de nada. Está totalmente amnésico. Ni siquiera sabe lo que es la tele.

- ¡Amnésico, oh pobrecito!

(Los visitantes)

Le dialogue évoque Eddie Barclay, chanteur puis producteur très connu en France, Stéphanie de Monaco (qui se lança dans la chanson en 1986, et qui était familièrement appelée Stéph'de Monac'à l'époque) et Michel Drucker (présentateur célèbre d'émissions de variétés). Ces références spécifiques à la culture populaire française, utilisées dans un but humoristique, ne sont généralement pas connues ailleurs. Elles donnent beaucoup de fil à retordre aux traducteurs. Aussi, les allusions à Eddie Barclay et à Michel Druker, personnages inconnus pour le grand public espagnol, ont été remplacées respectivement par productor et par tele. Par contre, comme Stéphanie de Monaco est ou a été connue du grand public en Espagne, l'ap- 
pellation familière a été adaptée et utilise la forme connue en Espagne (Stefi de Mónaco).

Cependant, les nombreuses références culturelles présentes dans les films ne correspondent pas toujours à un humour bateau. Parfois, elles représentent un humour beaucoup plus caustique, moins accessible d'emblée et plus difficile à comprendre par tous les spectateurs (exemple [16]).

(16) - Vas-y, appelle la police! Linda de Suza, va!

(La haine)

- Vamos llama a la policía si te da la gana vieja bruja, vete a tomar por culo.

(El odio)

Les trois jeunes protagonistes du film essaient de rendre visite à un ami de Saïd qui lui doit de l'argent. La concierge de l'édifice ne veut pas les laisser passer et les interpelle avec un accent portugais. Saïd l'apostrophe en l'appelant Linda de Suza, faisant ainsi référence au fait que, dans les années 60 et 70, nombre de Portugais et d'Espagnols immigrèrent en France et beaucoup y travaillèrent comme concierges. L'humour est sombre, teinté d'un racisme, jusqu'à un certain point paradoxal (Saïd est d'origine maghrébine et a un jeune Noir et un jeune Juif pour amis). La traduction de Linda de Suza par vieja bruja en espagnol, qui équivaut à vieille sorcière, fait perdre la référence culturelle ainsi que l'humour caustique. Dans les deux exemples, les références trop lointaines ont fait l'objet d'une naturalisation (domestication; Venuti 1995).

\subsubsection{Variations diastratiques et diatopiques}

L'accent, l'inflexion et le ton de voix, donc l'interprétation des acteurs, constituent les éléments clés de la manifestation de l'humour et donc de la transmission de la culture. Il est fréquent que ces marques n'apparaissent pas dans les versions doublées, ce qui constitue une grande perte, car les valeurs et les connotations qui leur sont associées disparaissent alors pour les spectateurs espagnols. Ainsi, dans Les visiteurs, les variantes diastratiques sont tournées en dérision dans de nombreuses scènes de façon à susciter la moquerie à l'égard des classes sociales. Les manières, les accents et les manies de langage sont représentatifs de toutes les classes sociales.

Dans les exemples (17) et (18), le facteur culturel est essentiellement lexical et phonétique (accent).

(17) - Non mais, non mais, lâchez-moi, mais, non mais attendez, vous m'étouffez, vous me faites hyper mal. Mais calmez-vous, mais, espèce de brute!

(Les visiteurs)

- ¡Déjeme, oiga me está asfixiando, me hace mucho daño, pero cálmese pedazo de bruto!

(Los visitantes)

(18) - Oui, eh ben vous irez sans moi, moi j’ai une matinée hyper chargée...

(Les visiteurs)

- Si, pero conmigo no contéis, tengo una mañana muy ocupada.

(Los visitantes)

Le facteur lexical ici correspond à l'emploi de certains mots typiques (hyper, hyper mal; hyper chargée), mais c'est surtout l'accent qui est caractéristique. Cependant, 
la version doublée ne rend aucun des deux en espagnol: le traducteur n'emploie pas ces mots typiques ce qui constitue encore une perte pour le public cible. En Espagne aussi, nous pouvons retrouver cette dichotomie et de nombreux humoristes ou films reproduisent également ces accents si particuliers. La version doublée en espagnol n'emploie pas ces mots clés qui existent également et les voix des doubleurs ne reproduisent pas ces accents caractéristiques. Or, les snobs espagnols (dits pijos) ont un accent particulier et emploient de nombreux préfixes du genre super. Le doublage aurait donc pu y avoir recours.

Les variations d'accent en fonction des régions ou des pays (diatopie) sont également un procédé humoristique. L'exemple (19), tiré du Dîner de cons, met en scène la victime, François Pignon, qui imite l'accent et les tournures belges.

(19) - Bonsoir [prononcé bonsouère] Monsieur Leblanc! Georges Van Brueghel à l'appareil. Pardon de vous déranger à une heure aussi tardive, je suis producteur belge, n'est-ce pas, j'arrive de Belgique une fois, et je suis très intéressé par votre roman... votre roman...

(Le dîner de cons)

- Ha ha, buenas noches señor Leblanc, soy Georges Van Brueghel, perdone que le llame a una hora tan intempestiva pero soy un productor belga ¿Sabe? Acabo de llegar de Bélgica y estoy muy interesado en su novela. Euh, ¿Como se llama?

(La cena de los idiotas)

Les particularités lexicales (n'est-ce pas, une fois) et l'accent belge exagéré dans la scène, font classiquement rire les Français. Dans la version espagnole, on a remplacé l'accent belge par un accent français qui peut parfois également faire rire les Espagnols. Il y a cependant une petite incohérence, car la traduction conserve les mots belga et Bélgica.

\subsubsection{Blagues et jeux de mots}

Il est parfois possible de traduire des blagues tout en préservant l'effet humoristique et sans que l'adaptation soit nécessaire, comme dans l'exemple (20):

(20) - Un con qui en moins d'une heure amène ta femme à l'adultère et toi au contrôle fiscal, c'est tout de même prodigieux!

(Le dîner de cons)

- En menos de una hora un idiota empuja tu mujer a cometer adulterio y a ti a las garras del fisco.

(La cena de los idiotas)

Par contre, les jeux de mots demandent généralement à être adaptés pour rendre l'effet comique (exemple [21]):

(21) - C'était votre sœur.

- J'ai pas de sœur.

- Vous n'avez pas de sœur? J’lui ai dit: «Qui est à l'appareil ?» Et elle m’a dit: «Sa sœur».

- Il a appelé Marlène.

- C'est pas votre sœur?

- C'est son nom Sasseur, Marlène Sasseur.

- J'pouvais pas savoir moi, elle me dit: «C'est Marlène, sa sœur».

(Le dîner de cons) 
- Era su hermana.

- No tengo ninguna.

- ¡No puede ser! He preguntado: «¿Con quién hablo?» Y me ha dicho: "Con su hermana».

- ¡Era Marlène!

- ¿Y no es su hermana?

- No, se llama Sermana, Marlène Sermana.

- Ouh, y ¿cómo quería que lo supiera? Me ha dicho soy Marlène su hermana,; es muy fácil confundirse!

(La cena de los idiotas)

\subsubsection{Humour à caractère sexuel}

L'humour connoté sexuellement, notamment en ce qui concerne l'infidélité, est fréquent partout. Se comprenant facilement dans tous les pays, il est très utilisé par les humoristes.

L'exemple (22) évoque une situation d'autant plus comique que Pierre Brochant entend la conversation entre sa victime, François Pignon, et son interlocuteur, l'inspecteur des impôts Cheval, ce qui lui confirme qu'il est trompé par sa femme.

(22) - C'est bien toi qui est sur le dossier Meneaux, Pascal Meneaux, le publicitaire?

- Affirmatif!

- Je sais qu'il a une garçonnière et je voudrais avoir l'adresse!

- Ah, t'es pas du tout son genre tu sais!

- Comment?

- Il les aime avec plus de poitrine et moins de poil aux pattes.

- C'est un boute-en-train! C'est sérieux Lucien, j’ai besoin de cette adresse, vite!

- Et pourquoi tu veux l'adresse du baisodrome de Meneaux?

- Je vais te dire la vérité, c'est pour un ami qui pense que sa femme est là-bas.

$-[\ldots]$

- Si je comprends! Eh, je l'ai vu à l'œuvre, le Meneaux... Dès qu'il y a un jupon à l'horizon, il devient fou...

(Le dîner de cons)

- ¿Eres tú el que lleva el caso de Pascal Meneaux el publicista?

- iEn efecto!

- Tiene un piso de soltero en París y quiero su dirección.

- ¡No creo que seas su tipo, hahaha!

- ¿Cómo?

- ¡Le gustan con más pecho y las piernas menos peludas, hahaha!

- ¡Es la monda, haha, hihihi! ¡No, en serio, necesito su dirección date prisa!

- ¿Para qué quieres la dirección del picadero de Meneaux?

- La verdad es que es para un amigo que cree que su mujer puede estar allí.

$-[\ldots]$

- ¡Ya lo creo! ;Hoho, yo he visto a Meneaux en acción, en cuanto ve una falda, se vuelve loco!

(La cena de los idiotas)

La situation deviendra encore plus loufoque lorsque l'inspecteur des impôts, archétype du personnage antipathique, apprend que c'est lui que sa femme trompe avec Meneaux. La traduction est ici toute naturelle et littérale et ne nécessite alors aucune adaptation particulière. 
Dans l'exemple (23), les trois protagonistes rebelles cherchent à draguer des jeunes filles dans l'exposition qu'ils n'apprécient pas (voir exemple [3]). Hubert va engager la conversation avec deux jeunes filles pour les présenter à Saïd. Il le fait assez facilement, car il se montre aimable et charmeur. Mais Saïd est trop direct et choque les jeunes filles. Finalement, la présence de Vinz, qui est le plus agressif des trois, fait qu'au lieu de draguer et de discuter gentiment, tout se termine en une altercation. La tension monte et Vinz demande à une des deux filles si elle se prend pour la fille du Wonderbra (une marque de sous-vêtements féminins). Le film utilise ici l'humour pour faire baisser la tension.

(23) - Excusez-moi les filles, mon nom c'est Hubert et mon copain, il est trop romantique et trop timide. C'est un poète, quoi, et en fait il aimerait trop discuter avec vous! C'est celui-là, là. Ouais, c'est possible, ouais?

$-[\ldots]$

- Non, mais il est super cool, il est trop gentil, le gars, le gars, il est trop gentil! C’est bon Saïd, ramène-toi!

$-[\ldots]$

- Salut, salut les filles, c'est moi, Saïd. [...]

- Il vous a dit que je vous trouvais bonne aussi?

- Bonne à quoi?

- À ton avis?

- On croyait que t'étais timide.

- Timide? Ouais, je suis timide, je suis un grand timide! T’as un numéro de téléphone pour qu'on se voit plus tard ou on se fait un petit cinoche?

$-[\ldots]$

- Faites chier, hein, vraiment... Nous, on veut bien parler avec vous, mais tout de suite, j'chais pas, vous êtes agressifs, comment vous voulez qu'on vous respecte?

- Et toi là, tu t'es pris pour qui ? La fille qui fait la pub pour Wonderbra ou quoi, là?...

(La haine)

- Disculpad chicas, me llamo Hubert y tengo un amigo que es muy romántico y muy tímido y, bueno vamos, le encantaría hablar con vosotras. Es ese, ...sí. ¿Sería posible?

$-[\ldots]$

- ¡Un chico estupendo os lo prometo! ¡Es estupendo! ¡Saïd ven!

$-[\ldots]$

- Hola chicas... yo soy Said...

- ¿Sabes que estás muy buena?

- ¿Buena para qué?

- ¿Tu que crees?

- ¿Creía que eras un tímido?

- ¿Tímido? ¡Si, soy tímido y muy tímido! ¿Tienes teléfono nos podríamos ver luego e ir al cine?

$-[\ldots]$

- ¡Sois unos pelmazos! ¿Queríamos hablar con vosotros pero ahora mismo os veo no sé muy agresivos! ¿Como queréis que os respetemos?

- ¿Tu quien te has creído que eres? ¡La chica que hace el anuncio del Wonderbra o qué!

(El odio)

Étant donné que les produits de marque Wonderbra sont commercialisés internationalement, l'adaptation n'est pas nécessaire. 


\section{Conclusion}

Le traducteur devrait être capable de comprendre toute l'information (linguistique et extralinguistique) présente dans les dialogues et les images d'un texte audiovisuel, car c'est de cette somme de signifiés que naîtra l'humour. Il devrait être également capable de transmettre cet humour dans la version traduite puis doublée ou du moins de produire le même effet humoristique. Cependant, les éléments linguistiques et extralinguistiques ne sont pas toujours transposables dans une autre langue ou culture.

Si nous résumons les résultats de l'analyse, nous observons qu'en aucun cas la version doublée ne produit un effet humoristique supérieur à celui de la version originale. Dans la première situation (exemples 1 à 3), l'expression uniquement visuelle de l'humour à travers les images n'entraîne pas de difficulté de traduction puisque la perception de l'humour est très similaire.

L'expression de l'humour à travers les images renforcées cette fois par un dialogue (exemples 4, 5, 6) s'appuie également sur l'aspect comique de certaines situations qui sont généralement plus ou moins transposables ou compréhensibles d'un pays à l'autre. Ceci sera généralement le cas, à l'exception de gestes, de conventions culturelles propres à un seul pays (Chaume 2004) ${ }^{16}$. Les images transmettent à elles seules le comique de la situation, cependant le dialogue renforce l'humour. La présence du dialogue implique une traduction qui sera selon le contenu et les langues concernées:

- totalement littérale;

- partiellement littérale avec un renforcement du caractère étranger de la production audiovisuelle;

- non littérale avec une naturalisation des références culturelles.

L'humour présent dans le texte et les dialogues est beaucoup plus recherché et complexe et sa réception en traduction est inférieure dans tous les cas, excepté dans les exemples (20), (22) et (23) qui utilisent un humour commun à toutes les sociétés (sexuel, économique) sans jeux de mots ni références culturelles. Les procédés adoptés sont:

- la traduction littérale dans le cas de jeux de mots ou blagues qui transmet le même genre d'humour;

- la traduction littérale qui fait perdre une partie du ou des sens;

- la traduction littérale qui oublie ou laisse de côté une phrase à contenu humoristique;

- la traduction qui ne tient pas compte du contenu diachronique de certains mots;

- la traduction-adaptation qui naturalise des contenus.

La présence de texte implique nécessairement la traduction. Selon les éléments linguistiques et extralinguistiques en présence, il peut ne pas y avoir de possibilité de recourir à une correspondance littérale, car celle-ci impliquerait automatiquement la non-compréhension de certains éléments par le public. Cette difficulté oblige alors le traducteur à rechercher une équivalence fonctionnelle (Nida 1964).

Dans les trois films analysés, le traducteur a adopté différentes solutions pour transférer ces éléments (traduction littérale, renforcement du caractère étranger du dialogue, naturalisation des références culturelles). Cependant, dans plus de $90 \%$ des 
cas où il n'y a pas de traduction littérale, la solution adoptée par le traducteur pour transférer ces éléments a été de les naturaliser. Le traducteur a donc réalisé une traduction communicative, car son objectif principal a été de reproduire le même effet ou, du moins, le plus similaire à l'original.

\section{NOTES}

1. Les visiteurs: Ils ne se sont pas nés d'hier (1993): Réalisé par Jean-Marie PoIré. Version espagnole: Los visitantes no nacieron ayer. Versus Entertainments (2009).

2. Le dîner de cons (1998): Réalisé par Francis Veber. Version espagnole: La cena de los idiotas. Manga films (2001).

3. La haine (1995): Réalisé par Mathieu Kassovitz. Version espagnole: El odio. Universal (2006).

4. Pour comprendre la traduction de l'humour dans les textes audiovisuels, il est nécessaire de connaître les facteurs caractéristiques de la traduction, d'une part, de l'humour, d'autre part, et des textes audiovisuels, par ailleurs.

5. Trois hommes et un couffin (1985): Réalisé par Coline SERREAU. Version espagnole: Tres hombres y un biberón.

6. Three Men and a Baby (1987): Réalisé par Leonard Nimoy. Version espagnole: Tres hombres y un bebé.

7. La cage aux folles (1987): Réalisé par Édouard Molinaro. Version espagnole: La jaula de las locas o pequeños vicios.

8. The Birdcage (1996): Réalisé par Mike Nichols. Version espagnole: Una jaula de grillos (littéralement: une cage de grillons). Le terme jaula de grillos désigne un endroit où règnent un grand désordre, de la confusion.

9. Rappelons ici que beaucoup de situations humoristiques s'appuient autant sur le support linguistique que sur l'interprétation gestuelle du personnage.

10. Les textes audiovisuels constituent un genre paradigmatique dans lequel l'information non verbale joue un rôle très important. Ce qui nous permet précisément de regrouper ces textes dans un genre paradigmatique, ou genre de genres, c'est que dans presque tous ces textes, le sens émane de l'interaction entre l'information verbale et l'information non verbale.

11. Le moqueur ridiculisé se retrouve dans El burlador de Sevilla de Tirso de Molina, La farce de maître Pathelin, Le Coq et le Renard de Jean de La Fontaine, etc.

12. Voir Le Grand Robert de la langue française (2005): Paris: Éditions Le Robert. Version CDROM.

13. Voir Grevisse, Maurice (1993): Le bon usage. De Boeck-Duculot: Bruxelles, 75-76.

14. Voir Dictionnaire.sensagent.com (2009): Réussite. Consultée le 6 janvier 2010, <http://dictionnaire. sensagent.com/r\%C3\%A9ussite/fr-fr/>; Mediadico.com (2010): Réussite. Consultée le 6 janvier 2010, <http://www.mediadico.com/dictionnaire/definition/Reussite/1>.

15. Voir Dictionnaire.reverso.net (2009): Brossoir. Consultée le 6 janvier 2010, <http://dictionnaire. reverso.net/francais-definition/brossoir>.

16. Par exemple, lorsque l'on dit, en Espagne, qu'une personne est pistonnée, on dit estar enchufado (branché sur le secteur) et on ajoute trifásico (triphasique) en faisant le geste ad hoc avec trois doigts. À Tunis, par contre, on se touche l'épaule pour montrer qu'on est porté par quelqu'un. Ces gestes ne seront pas compris dans d'autres cultures.

\section{RÉFÉRENCES}

Bernal Merino, Miguel Ángel (2002): La traducción audiovisual. Alicante: Publicaciones Universidad de Alicante.

Chaume, Frederic (2004): Cine y Traducción. Madrid: Cátedra.

Díaz Cintas, Jorge (2003): Teoría y práctica de la Subtitulación. Inglés-Español. Barcelona: Ariel.

Mogorrón, Pedro (sous presse) : La traduction audiovisuelle est-elle une traduction spécialisée? In: Salah MejRI, dir. La traduction des textes spécialisés: méthode et corpus. Publications du CERES, Série Linguistique. 13. Tunis: CERES. 
NidA, Eugene (1964): Toward a Science of Translating with Special referente to Principles and Procedures Envolved in Bible Translating. Leide: E.J.Brill.

Venuti, Lawrence (1995): The Translator's Invisibility. Londres: Routledge.

Zabalbeascoa, Patrick (2001): La traducción del humor en textos audiovisuales. In: Miguel Duro, dir. La traducción para el doblaje y la subtitulación. Madrid: Cátedra, 251-266. 